\title{
BMJ Open Association of sleep duration and quality with blood lipids: a systematic review and meta-analysis of prospective studies
}

\author{
Marlot Kruisbrink, ${ }^{1,2,3}$ Wendy Robertson, ${ }^{1}$ Chen $\mathrm{Ji}^{1}{ }^{1}$ Michelle A Miller, ${ }^{1}$ \\ Johanna M Geleijnse, ${ }^{4}$ Francesco P Cappuccio ${ }^{5}$
}

To cite: Kruisbrink M, Robertson W, Ji C, et al. Association of sleep duration and quality with blood lipids: a systematic review and meta-analysis of prospective studies. BMJ Open 2017;7:e018585. doi:10.1136/ bmjopen-2017-018585

\section{- Prepublication history and} additional material for this paper are available online. To view these files, please visit the journal (http://dx.doi.org/10. 1136/bmjopen-2017-018585).

Received 10 July 2017 Revised 9 November 2017 Accepted 9 November 2017

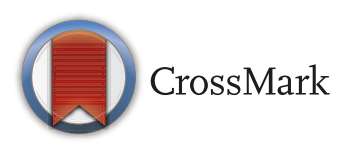

${ }^{1}$ Division of Health Sciences, Warwick Medical School, University of Warwick, Coventry, UK

${ }^{2}$ Department of Health Services Research, Faculty of Health, Medicine and Life Sciences, Maastricht University, Maastricht, The Netherlands ${ }^{3}$ CAPHRI, Maastricht University, Maastricht, The Netherlands ${ }^{4}$ Division of Human Nutrition, Wageningen University, Wageningen, The Netherlands ${ }^{5}$ ESH Centre of Excellence in Hypertension \& Cardiometabolic Research, University Hospitals Coventry \& Warwickshire NHS Trust, Coventry, UK

Correspondence to Professor Francesco

P Cappuccio;

f.p.cappuccio@warwick.ac.uk

\section{ABSTRACT}

Objectives To assess the longitudinal evidence of the relationships between sleep disturbances (of quantity and quality) and dyslipidaemia in the general population and to quantify such relationships.

Setting Systematic review and meta-analysis following the Preferred Reporting Items for Systematic Reviews and Meta-Analyses guidelines.

Methods We performed a systematic search of PubMed and Embase (up to 9 September 2017), complemented with manual searches, of prospective population studies describing the association between sleep duration and quality and the incidence of dyslipidaemias. Relative risks $(95 \% \mathrm{Cls})$ were extracted and pooled using a random effects model. Subgroup analyses by lipid type were performed. Heterogeneity and publication bias were also assessed. Quality was assessed with Downs and Black score.

Participants Studies were included if they were prospective, had measured sleep quantity and/or quality at baseline and either incident cases of dyslipidaemia or changes in blood lipid fractions assessed prospectively.

Primary outcome measures Incidence of dyslipidaemia and changes in lipid fractions. Dyslipidaemia was defined as a high total cholesterol, triglycerides, low-density lipoprotein cholesterol or low high-density lipoprotein cholesterol compared with the reference group.

Results Thirteen studies were identified (eight using sleep duration, four sleep quality and one both). There was heterogeneity in the sleep quality aspects and types of lipids assessed. Classification of sleep duration (per hour/groups) also varied widely. In the pooled analysis of sleep duration (6 studies, 16 cohort samples; 30033 participants; follow-up 2.6-10 years), short sleep was associated with a risk of $1.01(95 \% \mathrm{Cl} 0.93$ to 1.10$)$ of developing dyslipidaemia, with moderate heterogeneity $\left(I^{2}=56 \%, P=0.003\right)$ and publication bias $(P=0.035)$. Long sleep was associated with a risk of $0.98(95 \% \mathrm{Cl} 0.87$ to 1.10$)$ for dyslipidaemia, with heterogeneity $\left(I^{2}=63 \%\right.$, $\mathrm{P}<0.001)$ and no significant publication bias $(\mathrm{P}=0.248)$. Conclusion The present analysis was unable to find supportive evidence of a significant relationship between sleep duration and the development of dyslipidaemia. However, heterogeneity and small number of studies limit the interpretation.

PROSPERO registration number CRD42016045242.
Strengths and limitations of this study

- This is the first study evaluating the collective prospective evidence of the association between sleep duration and biomarkers of lipid metabolism.

- Strengths of this review include the broad search strategy and in-depth quality assessment of studies.

- Limitations to interpretation are: heterogeneity of exposure and outcome measurements and small number of studies.

- The results can only be representative of published and included studies.

\section{BACKGROUND}

Research into sleep and its effects on health has increased in recent years. This has been accompanied by public health concerns about the declining quality and quantity of sleep in modern society. ${ }^{1}$ Both short and long sleep duration are consistently associated with mortality and serious chronic diseases, such as diabetes and cardiovascular disease (CVD).$^{2-4}$ Similarly, poor sleep quality has been associated with mortality and CVD. ${ }^{45}$ CVD is the leading cause of non-communicable disease deaths globally and deaths by CVD have risen by $12.5 \%$ between 2005 and $2015 .{ }^{6}$ There is still debate about whether the association between sleep and CVD is causal or whether sleep disturbances are merely symptoms or risk markers of disease. ${ }^{7}$ Understanding the possible mechanisms through which sleep affects CVD can provide important supportive evidence for a causative link.

U-shaped relationships between duration of sleep and risk factors for CVD, such as hypertension and metabolic syndrome have been observed. ${ }^{89}$ For obesity, the longitudinal association is most clear in paediatric populations, in which shorter sleep is associated with an increased risk of obesity. ${ }^{10}$ Fewer studies have been performed on sleep quality, 


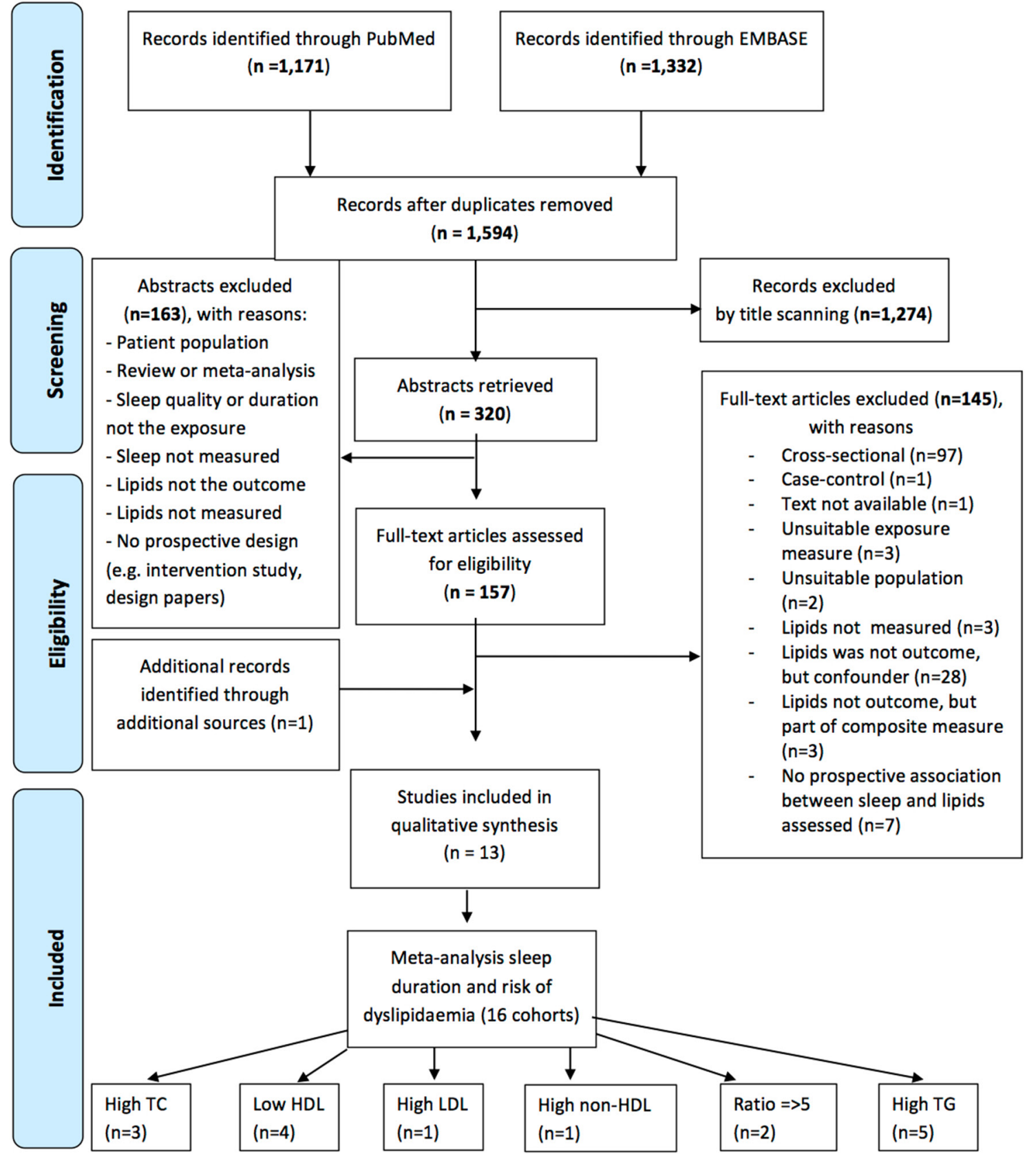

Figure 1 PRISMA flow diagram of study selection. HDL, high-density lipoprotein; LDL, low-density lipoprotein; PRISMA, Preferred Reporting Items for Systematic Reviews and Meta-Analyses; TC, total cholesterol; TG, triglyceride.

but poor sleep quality has also been associated with an increased risk of hypertension, ${ }^{11}$ metabolic syndrome ${ }^{12}$ and diabetes. ${ }^{2}$

An unfavourable blood lipid profile, including high total cholesterol (TC) and low-density lipoprotein cholesterol (LDL-C), is a well-established risk factor for CVD. ${ }^{13}$ Circulating lipids are influenced by lifestyle factors such as diet, smoking and physical activity. ${ }^{14}$ Whether sleep duration and quality are associated with blood lipids remains to be ascertained.

Systematic reviews of observational studies suggest a lack of consistency in the association between sleep duration and lipid profiles, with a large heterogeneity in the classification of exposure and outcome and the type of analysis. Furthermore, these were mainly based on cross-sectional evidence-hence unable to establish a temporal relationship between exposure and outcomeand did not evaluate sleep quality as a potential exposure of interest. ${ }^{15}{ }^{16}$ In recent years, new prospective studies that include measures on sleep and blood lipids have emerged. Nadeem et $a l^{17}$ performed a meta-analysis of 64 observational studies involving 18116 patients on obstructive sleep apnoea (OSA) and the blood lipid profile. They found that OSA was associated with a significantly higher risk of dyslipidaemia, for example, high TC and LDL-C, high triglyceride (TG) and low high-density lipoprotein cholesterol (HDL-C). However, this meta-analysis was performed in a specific patient group, did not include 
sleep duration as an exposure and was based on cross-sectional studies.

To the best of our knowledge, a meta-analysis of prospective studies on sleep quality and duration, and blood lipids in the general population without diagnosed sleep disorders has not yet been published. We set out to systematically evaluate prospective studies for an association between sleep duration and quality, and blood lipids in the general population and to pool the evidence in a meta-analysis.

\section{DATA AND METHODS}

This systematic review and meta-analysis was conducted according to the Preferred Reporting Items for Systematic Reviews and Meta-Analyses (PRISMA) guidelines. ${ }^{18}$ PROSPERO registration number: CRD42016045242, available from http://www.crd.york.ac.uk/PROSPERO/ display_record.asp?ID=CRD42016045242.

\section{Search strategy}

The electronic databases, PubMed (from 1996) and EMBASE (from 1947), were searched on 9 September 2017 using keywords related to exposure (sleep duration and quality), outcome (blood lipids) and design (prospective). Abbreviations, plural forms and alternate spellings (American-English) of keywords were searched. The search was restricted to human research and published journal articles. No language restriction was applied. In addition, a manual check of reference lists was performed using (1) previous review articles on the subject, (2) relevant review articles identified in the search and (3) articles included in the present study. Additional searches were performed into the studies that measured lipids at baseline and follow-up, but did not report on lipids, to see if additional publications were available which did report on the outcome of interest.

\section{Study selection}

After title and abstract scanning, full-text articles were retrieved. Prospective articles were evaluated for inclusion by two of three investigators (MK, WR and FPC) according to the following criteria set a priori: (A) original published article, (B) observational prospective design, (C) a baseline assessment of exposures (sleep duration or sleep quality) and (D) one of the following outcomes: (1) a change in serum lipids over time or (2) a relative risk of developing dyslipidaemia in short or long sleepers compared with the reference sleep category. Studies were excluded if (A) exposure was napping or shift work, (B) population had a diagnosed sleep disorder like OSAS or pre-existing cardiovascular or metabolic disease, (C) it was a case-control study. No sample size, age or duration of follow-up restriction was applied. Disagreement on inclusion was resolved by discussion and consensus among the three investigators. Authors were contacted for additional data.

\section{Data extraction}

Data from each study was extracted independently by two investigators (MK and FPC). Extracted data included: first author, year of publication, country of origin of the population, recruitment year of cohort, age (at sleep assessment), sex, duration of follow-up, number of participants included, methods of assessment of both exposure and outcome, definitions of sleep categories, relative risks $(\mathrm{RR}), \mathrm{HR}, \mathrm{OR}$, regression coefficients $(\beta)$ representing changes in lipid levels, 95\% CI, SE and adjustment for covariates. SEs were derived from CI if not reported (online supplementary appendix table A1). The most adjusted estimates were used for analysis. When data were reported for men and women separately, they were entered for analysis as two separate cohorts. When data from the same cohort was published in separate papers, only one estimate was used (usually the longer follow-up or the largest dataset). Differences in extracted information were resolved by discussion and consensus among two of the investigators.

\section{Risk of bias assessment}

The quality of the included studies was assessed using the Downs and Black Quality Index Score. ${ }^{19}$ This checklist includes items for measuring a study's reporting quality, external validity, bias, confounding and power. The maximum score for prospective studies is 20 .

\section{Statistical analysis}

A random effects model with inverse-variance weighting was used to pool HRs, ORs and RRs into RRs for developing high TC, low HDL-C, TC/HDL-C ratio $\geq 5$ and high TG in short sleepers and long sleepers compared with the reference category. Ratio measures and standard errors were transformed into natural logarithms for analysis. For a detailed overview and examples of data transformations performed, see online supplementary appendix table A2. Changes in lipid levels over time were meta-analysed using a random effects model when at least two cohorts with a similar exposure and outcome measurement were available. Due to heterogeneity in sleep quality aspects and types of outcomes reported, we were unable to metaanalyse the studies on sleep quality. Publication bias was assessed with examination of funnel plot symmetry and Egger's regression test for small study effects when the number of cohorts available was greater than 2. Heterogeneity was investigated with $Q$ test statistic and quantified by $\mathrm{I}^{2}$ statistics. The following thresholds for $\mathrm{I}^{2}$ interpretation from Cochrane Reviews were used: ' $0 \%$ to 40\%: might not be important; $30 \%$ to $60 \%$ : may represent moderate heterogeneity; $50 \%$ to $90 \%$ : may represent substantial heterogeneity; $75 \%$ to $100 \%$ : considerable heterogeneity'. ${ }^{20}$ The influence of individual studies was investigated by excluding one study at a time. A two-tailed $\mathrm{P}$ value $<0.05$ was considered statistically significant. Statistical analyses were performed with Stata V.14 (StataCorp, College Station, Texas, USA). 


\section{RESULTS}

\section{Identified studies}

Searches yielded 1594 titles (figure 1). After title and abstract scanning, 157 full-text articles were retrieved. Twelve studies were identified in the search, seven concerned only sleep duration, one concerned only sleep quality and one concerned both. Searching the references of included studies yielded one additional study regarding sleep quality, yielding a total of 13 studies. Four authors were contacted ${ }^{21-24}$ for additional data of whom one could provide data.

\section{Assessment and definition of exposures}

Sleep duration was mostly self-reported, either by questionnaire $^{2124-28}$ or interview ${ }^{29} 30$ (table 1). Three studies used accelerometry to assess sleep duration. ${ }^{23} 3132$ Sleep duration was analysed as a continuous measure in four studies, meaning a risk ${ }^{29}$ or change in lipid levels per hour of sleep increase ${ }^{23} 3132$ was reported. Two studies used qualitative groups ${ }^{27} 28$ and five used sleep duration groups for analysis. Short sleep was defined as $\leq 6 \mathrm{hou}$ rs, ${ }^{21}<5$ hours, ${ }^{24}<6$ hours ${ }^{25} 30$ and $<7$ hours. ${ }^{26}$ Long sleep duration was defined as $\geq 9$ hours, ${ }^{21}{ }^{25} \geq 7$ hours $^{24}$ and $\geq 10$ hours. ${ }^{2633}$ Subjective aspects of sleep quality that have been evaluated by questionnaire include difficulty falling asleep, ${ }^{33} 34$ difficulty maintaining sleep, ${ }^{33}$ unrefreshing or non-restorative sleep, ${ }^{33} 34$ presence or absence of sleep disorder, ${ }^{28}$ frequency of sleep duration ${ }^{27}$ and Pittsburgh Sleep Quality Index (PSQI) score. ${ }^{23}$ Sleep fragmentation was objectively assessed with accelerometry in one study. ${ }^{23}$

\section{Change from protocol}

In the original protocol submission to PROSPERO (CRD42016045242), the Outcome(s) section reads Primary outcomes: we expect most studies will have measured cholesterol. The expected primary outcomes are therefore changes in TC or the risk of developing hypercholesterolaemia. Secondary outcomes: the following outcomes will also be assessed: changes in serum levels HDL-C, LDL-C and TGs and the risk of developing dyslipidaemia (this can be hypercholesterolaemia, hypertriglyceridaemia, etc). The submission reflects the 'a priori' uncertainty on how the outcomes in prospective studies would look like. After the search, it became apparent that the most common form of outcome in prospective studies was indeed 'incidence of dyslipidaemia'. We report all outcomes originally planned to avoid the risk of selective outcome reporting.

\section{Assessment and definition of outcome}

For an overview of outcomes assessed, see table 1 . To assess outcomes, 10 studies used a fasting blood samples, ${ }^{21}$ 23-27 30-32 2 self-report ${ }^{28} 29$ and 1 data register. ${ }^{34}$ TC was assessed in six studies, ${ }^{2324} 26272932$ HDL-C in seven studies, ${ }^{23-26303133}$ LDL-C in three studies, ${ }^{2324}{ }^{26}$ TG in eight studies, ${ }^{21}$ 23-26 303133 non-HDL-C in one study ${ }^{24}$ and TC/ HDL-C ratio in one study. ${ }^{23}$ One study assessed changes in lipid levels, ${ }^{31} 10$ studies reported a risk of dyslipidaemia for one or more lipids or lipid fractions ${ }^{21} 24$ 25 27-33 and 1 study reported on both. ${ }^{23}$ Furthermore, one study assessed changes in lipid levels compared with a reference group. ${ }^{26}$ Dyslipidaemia was defined as a high TC, TG, LDL-C or low HDL-C compared with the reference group as described in table 1 .

\section{Study characteristics}

All identified publications were recent (20102017) (table 1). Ten studies were performed in adults, ${ }^{21}{ }^{23-28} 303334$ one in adolescents ${ }^{29} 32$ and one in children. ${ }^{31}$ Twelve studies recruited men and women, ${ }^{21} 23$ 25-34 four of these reported on outcomes in men and women separately. ${ }^{23} 25293234$ One study recruited only men. ${ }^{24}$ Follow-up ranged from 200 days to $>20$ years. Four studies were performed in the USA, ${ }^{23} 293233$ two in China ${ }^{2526}$ and Finland, ${ }^{32}{ }^{34}$ one in Canada, ${ }^{21}$ Denmark ${ }^{31}$, France, ${ }^{28}$ Japan $^{24}$ and South Korea. ${ }^{30}$

\section{Sleep quality}

In online supplementary appendix table A3, an overview of the results reported in the individual studies for sleep quality is given. In general, studies reported both favourable and unfavourable associations of poor sleep quality with blood lipids. The associations reported differed by lipid type and aspects of sleep quality assessed. Only Haaramo $e t a l^{34}$ reported significant associations. Those occasionally or frequently suffering from insomnia symptoms had a significantly increased risk of dyslipidaemia medication compared with those without insomnia symptoms.

\section{Sleep duration and dyslipidaemia risk}

The quality of studies included in the meta-analyses ranged from 12 to 18 out of a maximum score of 20 (see online supplementary appendix table A4). All studies scored high on items of reporting and bias. Studies scored less well on items of external validity and confounding. All studies lacked in adequate confounder adjustment by not adjusting for at least one of the following factors: baseline lipid levels, dyslipidaemia medication, other sleep variables or depression. Meta-analyses included three cohorts with high TC (21453 participants), four cohorts with low HDL-C (11851 participants), two cohorts with high TC/HDL-C ratio (503 participants) and five cohorts with high TG (11 450 participants). Meta-analyses of short sleep duration by different lipids fractions are shown in figure 2. In an overall pooled analysis of sleep duration (6 studies, 16 cohort samples; 30033 participants; follow-up 2.6-10 years), short sleep was associated with a risk of 1.01 (95\% CI 0.93 to 1.10$)$ of developing any dyslipidaemia, with moderate heterogeneity $\left(\mathrm{I}^{2}=56 \%\right.$, $\mathrm{P}=0.003)$ and publication bias $(\mathrm{P}=0.035)$. Short sleep was associated with a non-significant increased risk of developing high TC $(\mathrm{RR}=1.10 ; 95 \% \mathrm{CI} 0.99$ to $1.22 ; \mathrm{P}=0.07$; no heterogeneity and publication bias). There were not enough observations to perform an Egger's test for the risk of TC/HDL-C ratio $\geq 5$, there was no evidence for 


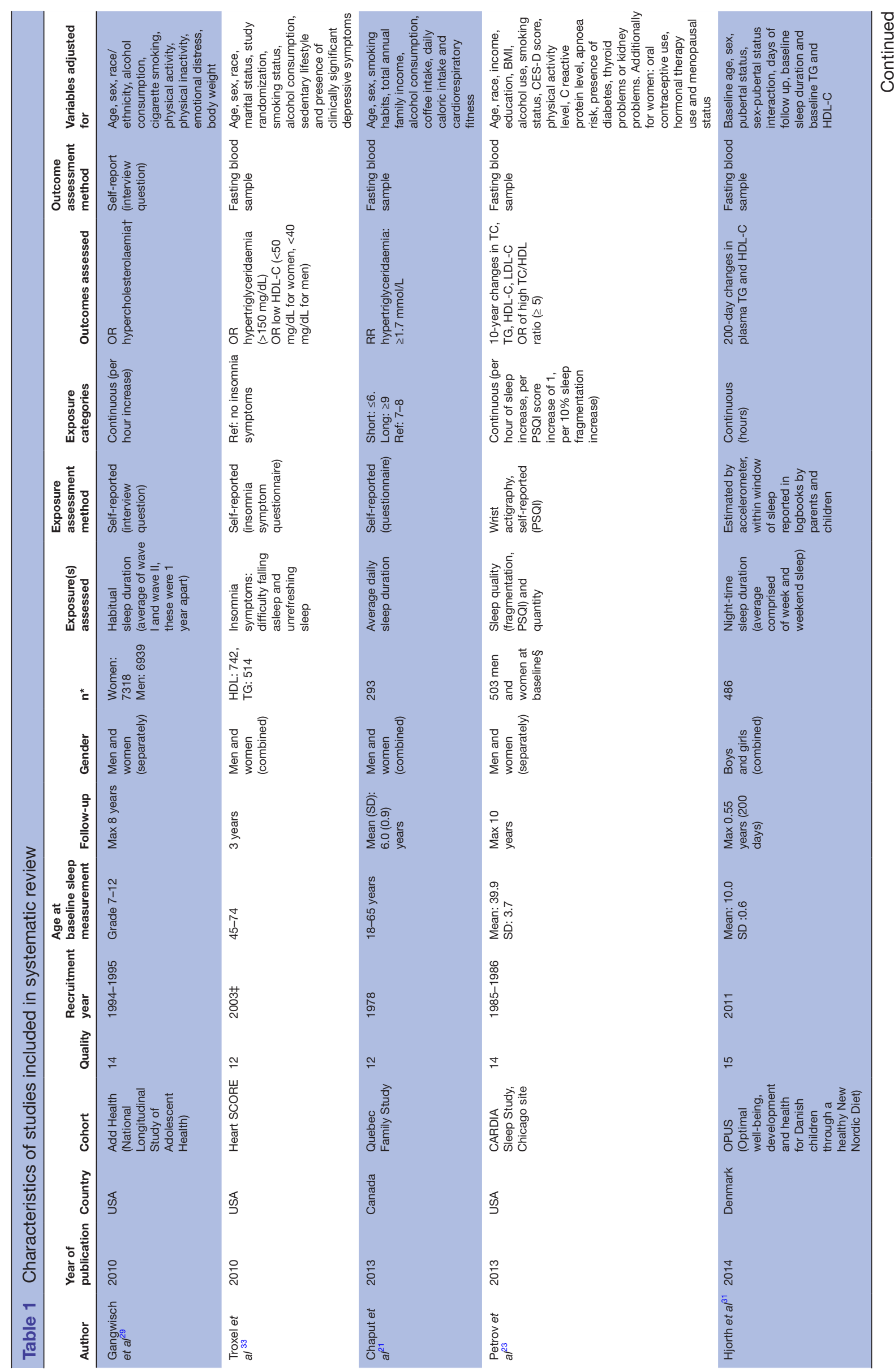




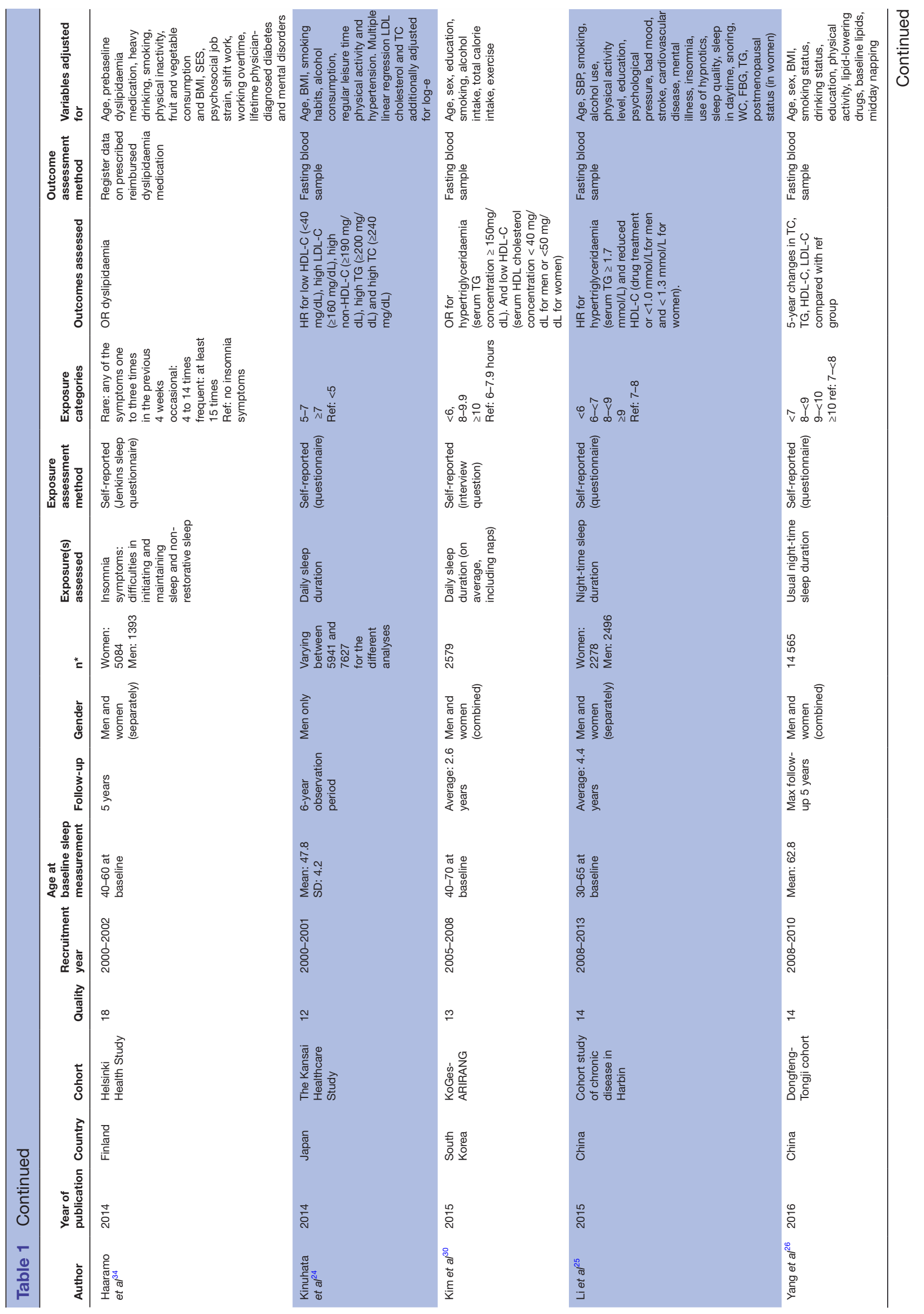




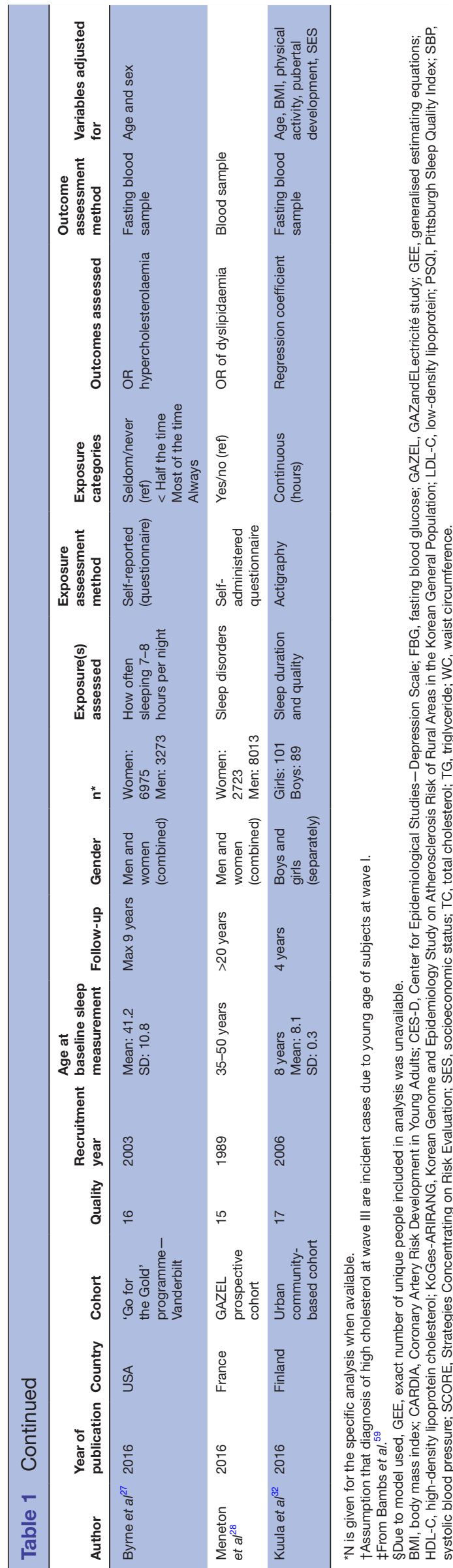

publication bias for the remaining lipid types (see online supplementary appendix figures A1 a-c).

Meta-analyses of long sleep duration by different lipid fractions are shown in figure 3. In an overall pooled analysis, the risk of any dyslipidaemia among long sleepers was 0.98 (95\% CI 0.87 to 1.10$)$, with heterogeneity $\left(\mathrm{I}^{2}=63 \%\right.$, $\mathrm{P}<0.001)$ and no significant publication bias $(\mathrm{P}=0.248)$. There were not enough observations to perform an Egger's test for the risk of TC/HDL-C ratio $\geq 5$, there was no evidence for publication bias for the remaining lipid types (see online supplementary appendix figure A1 d-f).

\section{Sleep duration and lipid changes over time}

There were too few studies to draw any meaningful conclusions from this analysis. (table 2). An increase in sleep duration was not associated with a change in HDL cholesterol. Furthermore, Yang $e t a l^{32}$ report changes in lipid levels in short and long sleepers compared with a 7-8hours reference group. None of these associations reached significance, except for an $0.085 \mathrm{mmol} / \mathrm{L}$ (95\% CI 0.014-0.156, P unreported) increase in TG for those sleeping $\geq 10$ hours compared with those sleeping $7-<8$ hours.

\section{DISCUSSION}

To our knowledge, this is the first systematic review and meta-analysis of the current prospective evidence on the relation between sleep quality, sleep duration and blood lipids in the general population. The results were not influenced by age, study quality, follow-up duration, gender or sleep assessment method. The analysis was carried out by separate lipid fractions. The risk of the development of dyslipidaemia varied among short and long sleepers and by lipid type.

Like Abreu et $a l,{ }^{16}$ we found that studies often adjusted for factors such as diet and body mass index, without exploring potential mediation, while the influence of other sleep variables are ignored. Sleep disordered breathing was not taken into account in any of the included studies, even though it has been associated with an increased risk of dyslipidaemia. ${ }^{18}$ Another factor that was inconsistently taken into account was stress, which can be a determinant of both poor sleep and increased stress levels. In the Whitehall II study, cortisol secretion was raised in those reporting short sleep duration and high sleep disturbance. ${ }^{35}$

Polysomnography is the gold standard for objective assessment of sleep quality and quantity, but objective sleep assessment is often not feasible in large cohort studies. Sleep quality and quantity were assessed subjectively by questionnaire or interview in most included studies. Moderate correlation between assessment of sleep duration by self-report and more objective actigraphy assessment have been observed in the Coronary Artery Risk Development in Young Adults study. ${ }^{36}$ Similarly, the Pittsburgh Sleep Quality Index and Epworth Sleepiness scale, two often used subjective measures of 


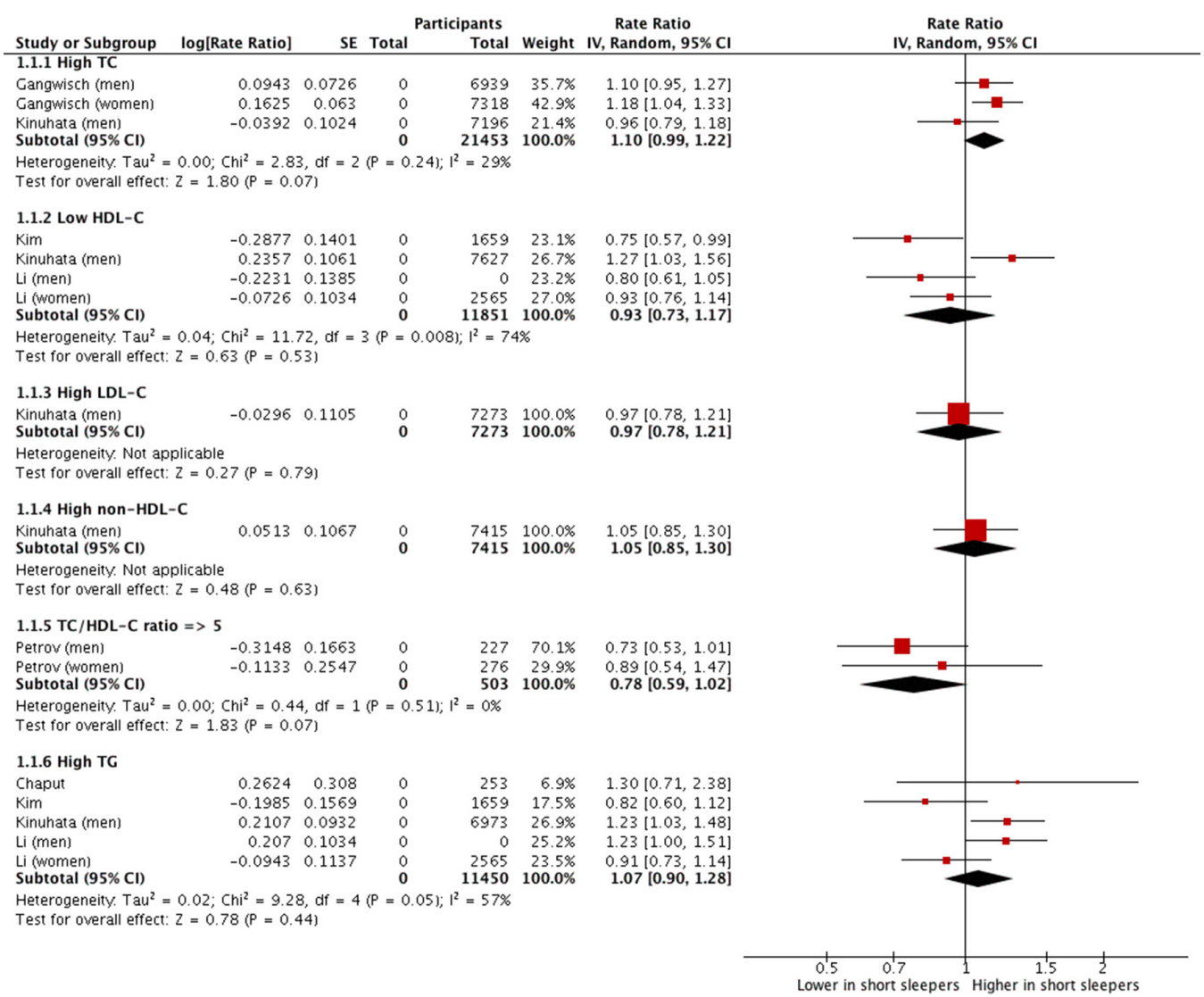

Figure 2 Forest plot of risk of dyslipidaemia in short sleepers. HDL-C, high-density lipoprotein cholesterol; LDL-C, low-density lipoprotein cholesterol; TC, total cholesterol; TG, triglyceride.

sleep quality, do not correlate well with objective sleep measures. ${ }^{37}$ In all included studies, a single measurement of sleep was taken at baseline, which may not represent the full sustained effect of sleep duration.

In previous systematic reviews, both long and short sleep duration were strongly associated with health outcomes, including cardiovascular disease. ${ }^{14}$ No such effects were found in this meta-analysis. It is possible that the effects of sleep duration on cardiovascular health are not mediated through blood lipids, ${ }^{38}$ but through other pathways such as obesity, hypertension and inflammation. ${ }^{1039-41}$ However, an effect of sleep duration on blood lipids would be biologically plausible. Sleep restriction is associated with an altered secretion of metabolic and hunger hormones, such as growth hormone, cortisol, leptin and ghrelin. ${ }^{42-44}$ Furthermore, sleep can influence eating behaviour and physical activity. Short sleep time and non-restorative sleep have been associated with a dietary alterations reflecting a higher intake of energy and fat. ${ }^{45-47}$ Sleep loss has also been shown to decrease physical activity in free-living conditions, ${ }^{48}$ and insufficient sleep could undermine dietary efforts to reduce adiposity. ${ }^{49}$ Several short-term experimental studies also suggest an effect of sleep restriction on blood lipid levels. ${ }^{50} 51$ Since it is difficult to have people sleep for long periods of time, mechanisms for the effects of long sleep duration on health have been less investigated and remain mostly speculative. It is possible that the observed relationship between long sleep duration and cardiovascular outcomes reflects long sleep duration being a risk marker or symptom of disease rather than a cause. ${ }^{7}$

\section{Strengths and limitations}

Strengths of this review include the broad search strategy and in-depth quality assessment of studies. The high heterogeneity of exposure and outcome measurements encountered in this review limited the scope of the meta-analysis. We were unable to perform a meta-analysis for sleep quality. The results can only be representative of published and included studies and the interpretation is limited by the small number of studies and some publication bias. Other limitations include the inability to directly adjust for confounding with study level meta-analysis and the fact that the quality of the meta-analysis cannot go beyond the quality of the included studies.

\section{Perspectives}

We do not yet have the strength of evidence needed to inform public health policy on the relation between sleep quality and duration and blood lipid profiles. In future research, individual patient data meta-analysis could provide possibilities to analyse data in a more homogeneous way. Furthermore, this review and meta-analysis focused on the general healthy population only. There are indications for an association between sleep and blood lipids in patients with diabetes ${ }^{52}$ and mental illness. ${ }^{53}$ 


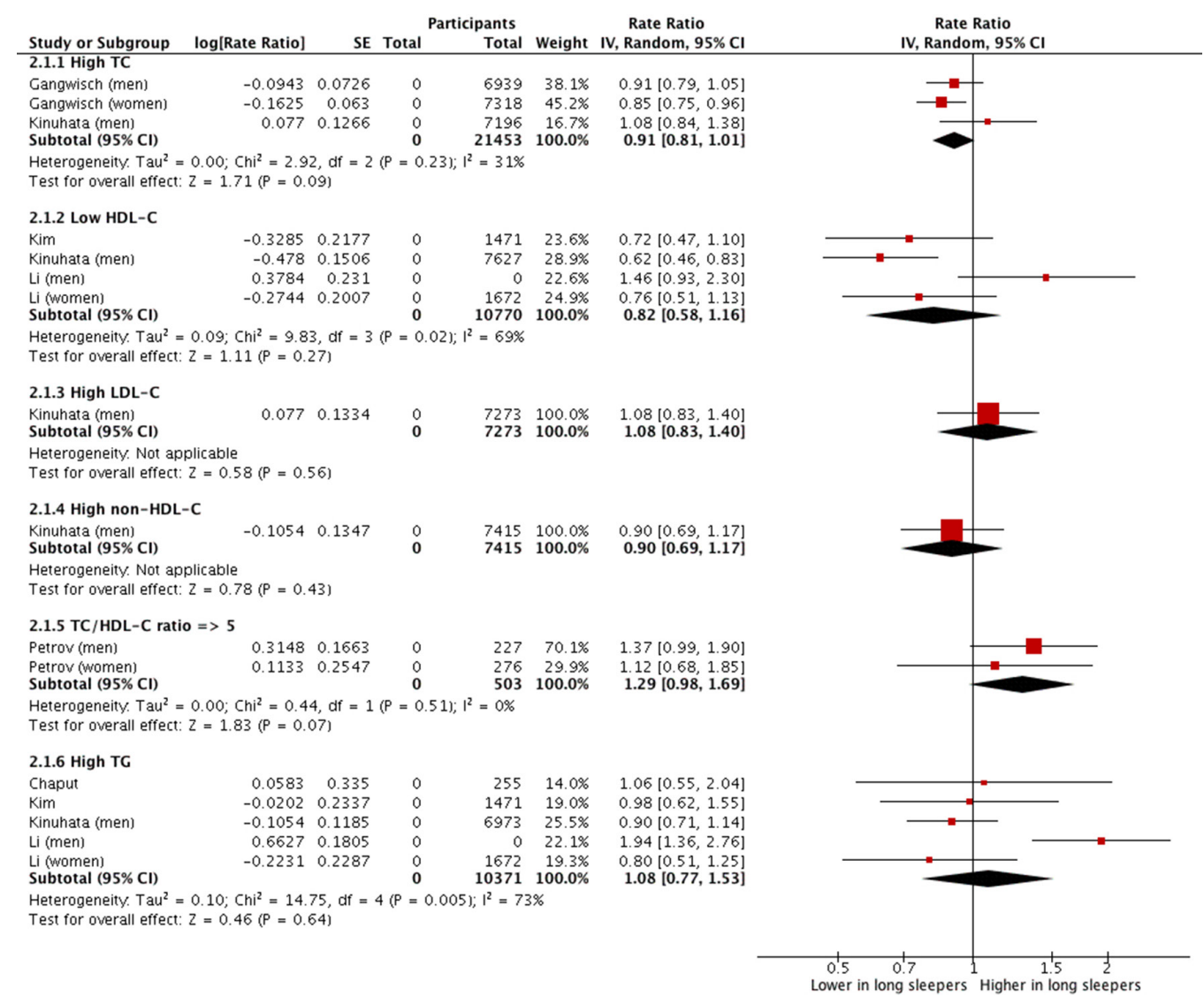

Figure 3 Forest plot of risk of dyslipidaemia in long sleepers. HDL-C, high-density lipoprotein cholesterol; LDL-C, low-density lipoprotein cholesterol; TC, total cholesterol; TG, triglyceride.

Other potential areas for future research are sleep timing and circadian disruption. Cross-sectional evidence indicates sleep timing and patterns may be associated with

Table 2 Meta-analytical results for continuous outcomes

\begin{tabular}{|c|c|c|c|c|}
\hline $\begin{array}{l}\text { Blood lipid } \\
\text { fraction }\end{array}$ & $\begin{array}{l}\text { No of } \\
\text { studies } \\
\text { and } \\
\text { cohorts }\end{array}$ & $\mathbf{n}$ & $\begin{array}{l}\text { Publication } \\
\text { bias }\end{array}$ & $\begin{array}{l}\text { Change in lipid } \\
\text { levels per hour } \\
\text { of sleep }\end{array}$ \\
\hline $\begin{array}{l}\text { Total } \\
\text { cholesterol }\end{array}$ & $\begin{array}{l}\text { One } \\
\text { study, } \\
\text { two } \\
\text { cohorts }\end{array}$ & 503 & - & $\begin{array}{l}0.14(0.06 \text { to } \\
0.23), P=0.001 \\
I^{2}=0.0\end{array}$ \\
\hline $\begin{array}{l}\text { HDL } \\
\text { cholesterol }\end{array}$ & $\begin{array}{l}\text { Two } \\
\text { studies, } \\
\text { three } \\
\text { cohorts }\end{array}$ & 989 & $P=0.020$ & $\begin{array}{l}0.00(-0.02 \text { to } \\
0.03) \\
P=0.719 \\
I^{2}=0.0\end{array}$ \\
\hline $\begin{array}{l}\text { LDL } \\
\text { cholesterol }\end{array}$ & $\begin{array}{l}\text { One } \\
\text { study, } \\
\text { two } \\
\text { cohorts }\end{array}$ & 503 & - & $\begin{array}{l}0.09(0.01 \text { to } \\
0.17) \\
P=0.033 \\
I^{2}=0.0\end{array}$ \\
\hline Triglycerides & $\begin{array}{l}\text { Two } \\
\text { studies, } \\
\text { three } \\
\text { cohorts }\end{array}$ & 989 & $P=0.450$ & $\begin{array}{l}0.01(0.01 \text { to } \\
0.01) \\
\mathrm{P}<0.001 \\
\mathrm{I}^{2}=0.0\end{array}$ \\
\hline
\end{tabular}

Values are reported in millimole per litre with $95 \% \mathrm{Cl}$.

HDL, high-density lipoprotein; LDL, low-density lipoprotein. unfavourable lipid profiles, ${ }^{54}$ although causality cannot be implied from those studies. Disruptions in the circadian rhythm have also been shown to be associated with metabolic alterations. ${ }^{55}$ Sleep disturbances are important to consider in the light of other CVD risk factors, such as obesity, hypertension and diabetes. randomised controlled trials that evaluate the effect of improved sleep habits on obesity and cardiovascular health are now becoming available. ${ }^{56-58}$

\section{CONCLUSION}

The present analysis was unable to find supportive evidence of a relationship between sleep duration and the development of dyslipidaemia. However, heterogeneity and small number of studies limit the interpretation. Further prospective studies are needed.

Acknowledgements We would like to thank JP Chaput for providing data on hypertriglyceridaemia in the Quebec Family Study.

Contributors MK set the search, reviewed part of the search output, extracted data, set up the database, drafted methods and results, contributed to analysis and discussion of results. WR contributed to the design of the search, reviewed part of the search output, contributed to the arbitration for data extraction, contributed to discussion of results. CJ carried out statistical analysis and contributed to the interpretation and discussion of results. MAM and JMG contributed to study design, interpretation and discussion of results. FPC developed the idea, contributed to study design, extracted data, supervised the analysis and drafted the final version 
of the manuscript. All authors contributed to, and approved, the final version of the manuscript. FPC in the guarantor.

Funding MK received an E Dekker student scholarship of the Dutch Heart Foundation.

Competing interests None declared.

Provenance and peer review Not commissioned; externally peer reviewed.

Data sharing statement № additional data are available.

Author note This study is part of the Sleep, Health and Society programme of the University of Warwick.

Open Access This is an Open Access article distributed in accordance with the Creative Commons Attribution Non Commercial (CC BY-NC 4.0) license, which permits others to distribute, remix, adapt, build upon this work non-commercially, and license their derivative works on different terms, provided the original work is properly cited and the use is non-commercial. See: http://creativecommons.org/ licenses/by-nc/4.0/

(c) Article author(s) (or their employer(s) unless otherwise stated in the text of the article) 2017. All rights reserved. No commercial use is permitted unless otherwise expressly granted.

\section{REFERENCES}

1. Cappuccio F, Miller MA, Lockley SW. Sleep, health, and society: the contribution of epidemiology, in sleep, health, and society: from aetiology to public health. USA: Oxford University Press, 2010.

2. Cappuccio FP, D'Elia L, Strazzullo P, et al. Quantity and quality of sleep and incidence of type 2 diabetes: a systematic review and meta-analysis. Diabetes Care 2010;33:414-20.

3. Cappuccio FP, D'Elia L, Strazzullo P, et al. Sleep duration and all-cause mortality: a systematic review and meta-analysis of prospective studies. Sleep 2010;33:585-92.

4. Cappuccio FP, Cooper D, D'Elia L, et al. Sleep duration predicts cardiovascular outcomes: a systematic review and meta-analysis of prospective studies. Eur Heart J 2011;32:1484-92.

5. Mallon L, Broman JE, Hetta J. Sleep complaints predict coronary artery disease mortality in males: a 12-year follow-up study of a middle-aged Swedish population. J Intern Med 2002;251:207-16.

6. Wang $\mathrm{H}$, Naghavi $\mathrm{M}$, Allen $\mathrm{C}$, et al. Global, regional, and national life expectancy, all-cause mortality, and cause-specific mortality for 249 causes of death, 1980-2015: a systematic analysis for the Global Burden of Disease Study 2015. Lancet 2016;388:1459-544.

7. Cappuccio FP, Miller MA. Sleep and mortality: cause, consequence, or symptom? Sleep Med 2013;14:587-8.

8. Gottlieb DJ, Redline S, Nieto FJ, et al. Association of usual sleep duration with hypertension: the Sleep Heart Health Study. Sleep 2006;29:1009-14.

9. Hall MH, Muldoon MF, Jennings JR, et al. Self-reported sleep duration is associated with the metabolic syndrome in midlife adults. Sleep 2008;31:635-43.

10. Wu Y, Gong Q, Zou Z, et al. Short sleep duration and obesity among children: A systematic review and meta-analysis of prospective studies. Obes Res Clin Pract 2017;11:140-50.

11. Liu RQ, Qian Z, Trevathan E, et al. Poor sleep quality associated with high risk of hypertension and elevated blood pressure in China: results from a large population-based study. Hypertens Res 2016;39:54-9.

12. Schmid SM, Schultes B. [Disturbed sleep as risk factor for metabolic syndrome]. Internist 2011;52:383-8.

13. World Heart Federation. Cardiovascular disease risk factors : cholesterol. http://www.world-heart-federation.org/cardiovascularhealth/cardiovascular-disease-risk-factors/cholesterol/ (accessed 7 Jul 2017).

14. National Health Service. High cholesterol. 2015 http://www.nhs.uk/ conditions/Cholesterol/Pages/Introduction.aspx (accessed 7 Jul 2017).

15. Abreu GA, Barufaldi LA, Bloch KV, et al. A systematic review on sleep duration and dyslipidemia in adolescents: understanding inconsistencies. Arq Bras Cardiol 2015;105:418-25.

16. Araghi $\mathrm{MH}$, Thomas GN, Taheri S. The potential impact of sleep duration on lipid biomarkers of cardiovascular disease. Clin Lipidol 2012;7:443-53.

17. Nadeem R, Singh M, Nida M, et al. Effect of obstructive sleep apnea hypopnea syndrome on lipid profile: a meta-regression analysis. J Clin Sleep Med 2014;10:475-89.
18. Moher D, Liberati A, Tetzlaff J, et al. Preferred reporting items for systematic reviews and meta-analyses: the PRISMA statement. Int J Surg 2010;8:336-41.

19. Downs SH, Black N. The feasibility of creating a checklist for the assessment of the methodological quality both of randomised and non-randomised studies of health care interventions. J Epidemiol Community Health 1998;52:377-84.

20. Cochrane Reviews. Identifying and measuring heterogeneity. http://handbook.cochrane.org/chapter_9/9_5_2_identifying_and measuring_heterogeneity.htm (accessed 7 Jul 2017).

21. Chaput JP, McNeil J, Després JP, et al. Short sleep duration as a risk factor for the development of the metabolic syndrome in adults. Prev Med 2013;57:872-7

22. Henderson M, Manousaki D, Radji S, et al. Turn off and Turn in: the influence of television viewing and sleep on lipid profiles in children. ESPE Abstracts 2015;84:3-841.

23. Petrov ME, Kim Y, Lauderdale D, et al. Longitudinal associations between objective sleep and lipids: the CARDIA study. Sleep 2013;36:1587-95.

24. Kinuhata S, Hayashi T, Sato KK, et al. Sleep duration and the risk of future lipid profile abnormalities in middle-aged men: the kansai healthcare study. Sleep Med 2014;15:1379-85.

25. Li X, Lin L, Lv L, et al. U-shaped relationships between sleep duration and metabolic syndrome and metabolic syndrome components in males: a prospective cohort study. Sleep Med 2015;16:949-54.

26. Yang L, Yang H, He M, et al. Longer sleep duration and midday napping are associated with a higher risk of CHD incidence in middle-aged and older chinese: the dongfeng-tongji cohort study. Sleep 2016;39:645-52

27. Byrne DW, Rolando LA, Aliyu MH, et al. Modifiable healthy lifestyle behaviors: 10-year health outcomes from a health promotion program. Am J Prev Med 2016;51:1027-37.

28. Meneton $\mathrm{P}$, Lemogne $\mathrm{C}$, Herquelot $\mathrm{E}$, et al. A global view of the relationships between the main behavioural and clinical cardiovascular risk factors in the GAZEL prospective cohort. PLoS One 2016;11:e0162386.

29. Gangwisch JE, Malaspina D, Babiss LA, et al. Short sleep duration as a risk factor for hypercholesterolemia: analyses of the national longitudinal study of adolescent health. Sleep 2010;33:956-61.

30. Kim JY, Yadav D, Ahn SV, et al. A prospective study of total sleep duration and incident metabolic syndrome: the ARIRANG study. Sleep Med 2015;16:1511-5.

31. Hjorth MF, Chaput JP, Damsgaard CT, et al. Low physical activity level and short sleep duration are associated with an increased cardio-metabolic risk profile: a longitudinal study in 8-11 year old Danish children. PLoS One 2014;9:e104677.

32. Kuula L, Pesonen AK, Kajantie E, et al. Sleep and lipid profile during transition from childhood to adolescence. J Pediatr 2016;177:173-8.

33. Troxel WM, Buysse DJ, Matthews KA, et al. Sleep symptoms predict the development of the metabolic syndrome. Sleep 2010;33:1633-40.

34. Haaramo P, Rahkonen O, Hublin C, et al. Insomnia symptoms and subsequent cardiovascular medication: a register-linked follow-up study among middle-aged employees. J Sleep Res 2014;23:283-91.

35. Kumari M, Badrick E, Ferrie J, et al. Self-reported sleep duration and sleep disturbance are independently associated with cortisol secretion in the Whitehall II study. J Clin Endocrinol Metab 2009;94:4801-9.

36. Lauderdale DS, Knutson KL, Yan LL, et al. Sleep duration: how well do self-reports reflect objective measures? The CARDIA Sleep Study. Epidemiology 2008;19:838-45.

37. Buysse DJ, Hall ML, Strollo PJ, et al. Relationships between the Pittsburgh Sleep Quality Index (PSQI), Epworth Sleepiness Scale (ESS), and clinical/polysomnographic measures in a community sample. J Clin Sleep Med 2008;4:563-71.

38. Matthews KA, Pantesco EJ. Sleep characteristics and cardiovascular risk in children and adolescents: an enumerative review. Sleep Med 2016;18:36-49.

39. Gangwisch JE, Feskanich D, Malaspina D, et al. Sleep duration and risk for hypertension in women: results from the nurses' health study. Am J Hypertens 2013;26:903-11.

40. Ferrie JE, Kivimäki M, Akbaraly TN, et al. Associations between change in sleep duration and inflammation: findings on C-reactive protein and interleukin 6 in the Whitehall II Study. Am J Epidemiol 2013;178:956-61.

41. Irwin MR, Olmstead R, Carroll JE. Sleep disturbance, sleep duration, and inflammation: a systematic review and meta-analysis of cohort studies and experimental sleep deprivation. Biol Psychiatry 2016;80:40-52

42. Spiegel K, Leproult R, L'hermite-Balériaux M, et al. Leptin levels are dependent on sleep duration: relationships with sympathovagal 
balance, carbohydrate regulation, cortisol, and thyrotropin. J Clin Endocrinol Metab 2004;89:5762-71.

43. Schmid SM, Hallschmid M, Jauch-Chara K, et al. A single night of sleep deprivation increases ghrelin levels and feelings of hunger in normal-weight healthy men. J Sleep Res 2008;17:331-4.

44. Broussard J, Brady MJ. The impact of sleep disturbances on adipocyte function and lipid metabolism. Best Pract Res Clin Endocrinol Metab 2010;24:763-73.

45. Corgosinho FC, Damaso AR, Ganen AP, et al. Short sleep time increases lipid intake in obese adolescents. Sleep Science 2013;6:26-31.

46. St-Onge MP, Roberts AL, Chen J, et al. Short sleep duration increases energy intakes but does not change energy expenditure in normal-weight individuals. Am J Clin Nutr 2011;94:410-6.

47. Grandner MA, Jackson N, Gerstner JR, et al. Sleep symptoms associated with intake of specific dietary nutrients. J Sleep Res 2014;23:22-34.

48. Schmid SM, Hallschmid M, Jauch-Chara K, et al. Short-term sleep loss decreases physical activity under free-living conditions but does not increase food intake under time-deprived laboratory conditions in healthy men. Am J Clin Nutr 2009;90:1476-82.

49. Nedeltcheva AV, Kilkus JM, Imperial J, et al. Insufficient sleep undermines dietary efforts to reduce adiposity. Ann Intern Med 2010;153:435-41.

50. Kerkhofs M, Boudjeltia KZ, Stenuit P, et al. Sleep restriction increases blood neutrophils, total cholesterol and low density lipoprotein cholesterol in postmenopausal women: A preliminary study. Maturitas 2007;56:212-5.

51. Vondra K, Brodan V, Dobiásová M, et al. Effect of sleep deprivation on cholesterol metabolism and triglyceridaemia in male volunteers. Eur J Appl Physiol Occup Physiol 1986;55:83-7.

52. Cooper AJ, Westgate K, Brage S, et al. Sleep duration and cardiometabolic risk factors among individuals with type 2 diabetes. Sleep Med 2015;16:119-25.

53. Soreca I, Wallace ML, Frank E, et al. Sleep duration is associated with dyslipidemia in patients with bipolar disorder in clinical remission. J Affect Disord 2012;141:484-7.

54. Lee S, Shin C. Habitual sleep initiation time and metabolic syndrome in middle-aged and elderly adults. Sleep Biol Rhythms 2015;13:371-9.

55. Machado RM, Koike MK. Circadian rhythm, sleep pattern, and metabolic consequences: an overview on cardiovascular risk factors. Horm Mol Biol Clin Investig 2014;18:47-52.

56. Cizza G, Marincola P, Mattingly M, et al. Treatment of obesity with extension of sleep duration: a randomized, prospective, controlled trial. Clin Trials 2010;7:274-85

57. Haack M, Serrador J, Cohen D, et al. Increasing sleep duration to lower beat-to-beat blood pressure: a pilot study. $J$ Sleep Res 2013;22:295-304.

58. Waloszek JM, Schwartz O, Simmons JG, et al. The SENSE Study (Sleep and Education: learning New Skills Early): a community cognitive-behavioural therapy and mindfulness-based sleep intervention to prevent depression and improve cardiac health in adolescence. BMC Psychol 2015;3:39.

59. Bambs C, Kip KE, Dinga A, et al. Low prevalence of "ideal cardiovascular health" in a community-based population: the heart strategies concentrating on risk evaluation (Heart SCORE) study. Circulation 2011;123:850-7. 